Brief note

\title{
Formation mechanism of Y-junctions in arrays of multi-walled carbon nanotubes
}

\author{
Dario Zilli ${ }^{\mathrm{a}}$, Silvia Blacher ${ }^{\mathrm{b}}$, Ana Lea Cukierman ${ }^{\mathrm{a}, \mathrm{c}}$, \\ Jean-Paul Pirard ${ }^{\mathrm{b}}$, Cedric J. Gommes ${ }^{\mathrm{b}, *}$ \\ a PINMATE - Depto Industrias, Facultad de Ciencias Exactas y Naturales, Universidad de Buenos Aires, \\ Int., Güiraldes 2620, (C1428BGA) Buenos Aires, Argentina \\ ${ }^{\mathrm{b}}$ Department of Chemical Engineering, University of Liège, B6a, Allée du 6 août 3, B-4000 Liège, Belgium \\ c Cátedra Farmacotecnia II, Depto Tecnología Farmacéutica, Facultad de Farmacia y Bioquímica, Universidad de Buenos Aires, Junín 956, (C1113AAD) Buenos Aires, Argentina
}

\section{A R T I C L E I N F O}

\section{Article history:}

Received 25 March 2008

Received in revised form 23 May 2008

Accepted 26 May 2008

Available online 6 June 2008

\section{Keywords:}

Carbon nanotubes

Y-junctions

Catalytic chemical vapor deposition

Transmission electron microscopy

\begin{abstract}
A B S T R A C T
Transmission electron microscopy of arrays of multi-walled carbon nanotubes reveals the presence of numerous Y-junctions. The diameter distributions of the samples are found to be bimodal, with one mode corresponding to the branches and the other mode to the trunk of the Y-junctions. The analysis of the micrographs shows that the junctions form via the merger of metal particles during the tip-growth of the tubes.
\end{abstract}

(c) 2008 Elsevier B.V. All rights reserved.

\section{Introduction}

Soon after their rediscovery in the nineties [1], carbon nanotubes (CNTs) were foreseen to be promising materials for electronic applications. Both theoretical and experimental studies were devoted to the electrical properties of individual single-walled, multi-walled, as well as double-walled CNTs [2,3]. In some instances, CNTs are naturally synthesized with junctions, some having unique electrical properties. For instance, Y-junctions of CNTs [4-7] can be used as nanometer-sized transistors [8]. Other types of junctions - like T-junctions, or $\mathrm{H}$-junctions [9] - could also be useful in the prospect of organizing CNTs into complex networks to make nano-electronics circuits [2].

The present work reports the large-scale occurrence of Yjunctions in multi-walled carbon nanotube arrays synthesized by catalytic chemical vapor deposition of iron phtalocyanine $[10,11]$. To the best of our knowledge, the growing of CNTs with Y-junctions by this particular synthesis process has not been reported previously. Furthermore, TEM characterizations

\footnotetext{
* Corresponding author.

E-mail address: Cedric.gommes@ulg.ac.be (C.J. Gommes).
}

give hints on the mechanism by which the observed Y-junctions form.

\section{Experimental}

Arrays of carbon nanotubes are synthesized by catalytic chemical vapor deposition (CCVD) under flowing $\mathrm{Ar} / \mathrm{H}_{2}$, by using analytical grade iron(II) phtalocyanine $\left(\mathrm{C}_{32} \mathrm{H}_{16} \mathrm{~N}_{8} \mathrm{Fe}\right)(99.99 \%$, Sigma-Aldrich) as precursor. The experimental procedure is similar to that reported previously [10-12]: the synthesis device consists in a semi-continuous horizontal tubular reactor ( $22 \mathrm{~mm}$ inner diameter, $1 \mathrm{~m}$ length), built in quartz. The reactor is externally heated by a two-zone furnace controlled by independent programmable temperature controllers, which provides control of the temperature and heating time.

The CNT arrays discussed in the present work were obtained for different sets of operating conditions. Experiments were carried out at reaction temperatures between 880 and $1050{ }^{\circ} \mathrm{C}$, under total gas flow rate $(F)$ between 10 and $100 \mathrm{ml}(\mathrm{STP}) / \mathrm{min}, \mathrm{H}_{2}$ molar fractions $\left(\left[\mathrm{H}_{2}\right]\right)$ between 0.25 and 0.66 , and total reaction time $(t)$ of 60 and $150 \mathrm{~min}$. CNTs were deposited on the reactor walls and on quartz substrates conveniently placed in the reactor. They were separated by scratching the surfaces of the walls or substrates. Four samples, labeled CNT-1 to CNT- 4 are analyzed in the 
Table 1

Synthesis conditions of the samples

\begin{tabular}{lrccc}
\hline Sample & $T\left({ }^{\circ} \mathrm{C}\right)$ & $F(\mathrm{ml} \mathrm{STP} / \mathrm{min})$ & {$\left[\mathrm{H}_{2}\right]$} & $t(\mathrm{~min})$ \\
\hline CNT-1 & 1050 & 10 & 0.66 & 60 \\
CNT-2 & 880 & 30 & 0.50 & 150 \\
CNT-3 & 880 & 100 & 0.66 & 60 \\
CNT-4 & 960 & 10 & 0.25 & 60 \\
\hline
\end{tabular}

$T$ : temperature; $F$ : total gas flow rate; $\left[\mathrm{H}_{2}\right]$ : molar fraction of hydrogen in the gas flow, $t$ : total reaction time.

present work, the synthesis conditions of which are reported in Table 1.

The samples were dispersed in ethanol in a sonication bath. A drop of the solution was deposited on carbon-coated copper microscopy grid, and evaporated. The micrographs were obtained on a Siemens Elmiskop 102 microscope, operated at $100 \mathrm{kV}$, at a magnification between $25,000 \times$ and $90,000 \times$. The CNTs' diameter distributions were obtained by measuring manually all the nanotubes visible in about 25 TEM images per sample, which is about 150 CNTs segments per sample. In the statistics, the data are weighed by the length of the measured segment: therefore, the distributions are to be interpreted as a density of the nanotubes' length as a function of their diameter [13]. The density of nanotube's length is estimated through Parzen's method [14] as

$L(d)=\frac{\left.\sum_{i=1}^{N} L_{i} G\left(d-d_{i}\right) / \sigma\right)}{\sum_{i=1}^{N} L_{i}}$

where $G$ is a Gaussian kernel, $d_{i}$ is the diameter of the $i$ th measured nanotube, $L_{i}$ is the length of the corresponding segment, and $\sigma$ is the error on the measurement of a single nanotube $(\sigma \approx 2 \mathrm{~nm})$. The latter value was estimated by measuring the outer diameter at various positions along the same nanotube; it was found to be independent of the diameter of the nanotube. For each sample, about 150 nanotubes were measured, which amounts for a total length of

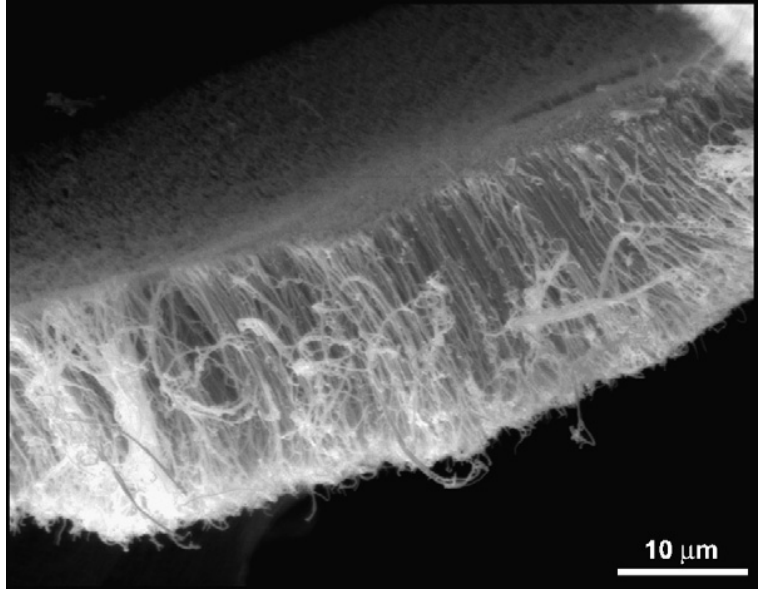

Fig. 1. Typical SEM picture of the pristine carbon nanotubes (sample CNT-1).

about $100 \mu \mathrm{m}$. Using Eq. (1) the diameter distribution is expressed as a sum of Gaussian distributions, without having to bin the data into user-defined classes [e.g. [15]].

\section{Results and discussion}

Fig. 1 shows a SEM micrograph of sample CNT-1; it is representative of all samples analyzed in the present work. Fig. 2a-d displays four TEM micrographs showing the presence of Y-junctions in the samples synthesized for different operating conditions. In these junctions, two thin nanotubes are merged into a thicker nanotube. Often, a metal particle is trapped at the junction (Fig. 2d), or close to it in one of the smaller branch (Fig. 2a).

The diameter distributions of samples CNT-2 and CNT-3 (corresponding to Fig. $2 \mathrm{~b}$ and $\mathrm{c}$ ) are plotted in Fig. $3 \mathrm{a}$ and b, respectively. The solid line in the figure has been obtained from Eq. (1), which
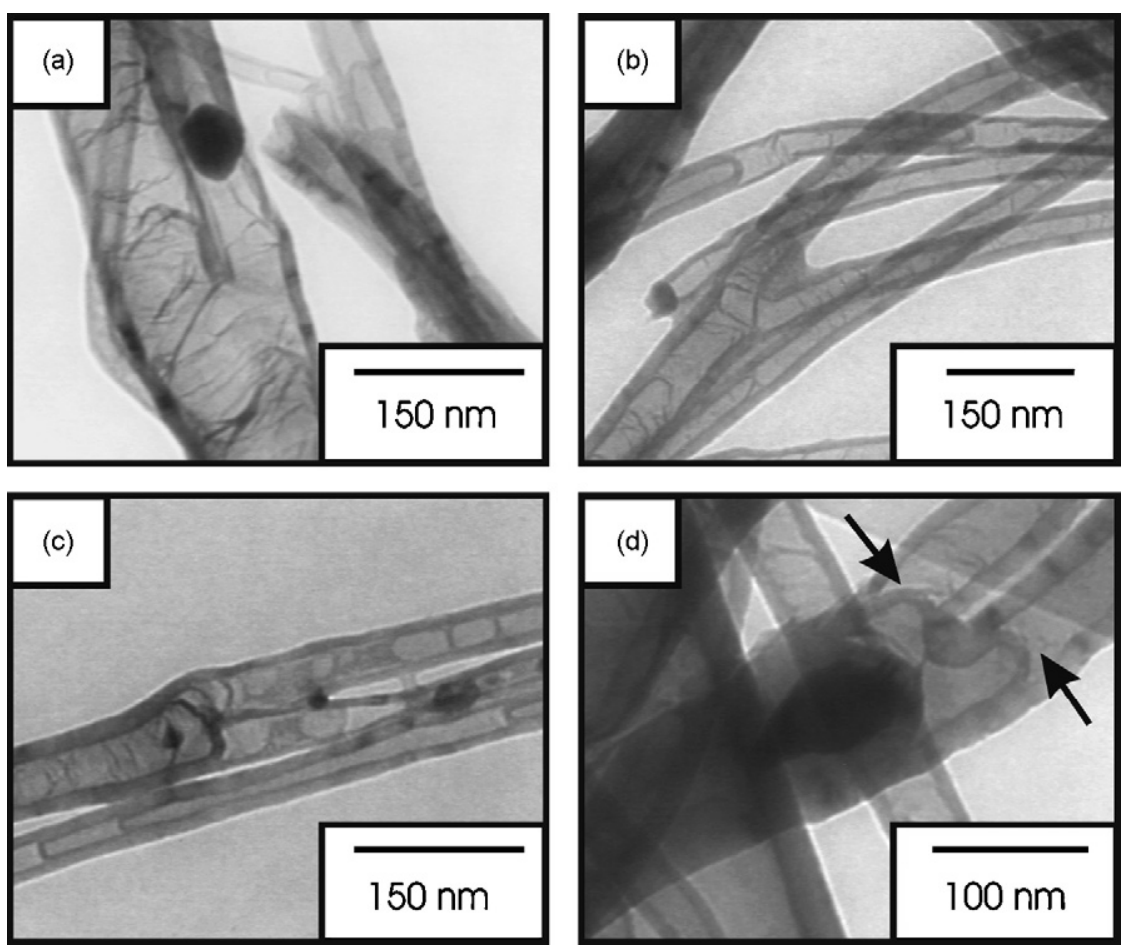

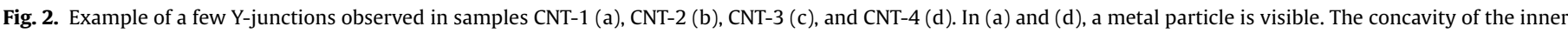
structures in (d) (arrows) gives hints on the way in which these junctions form. 

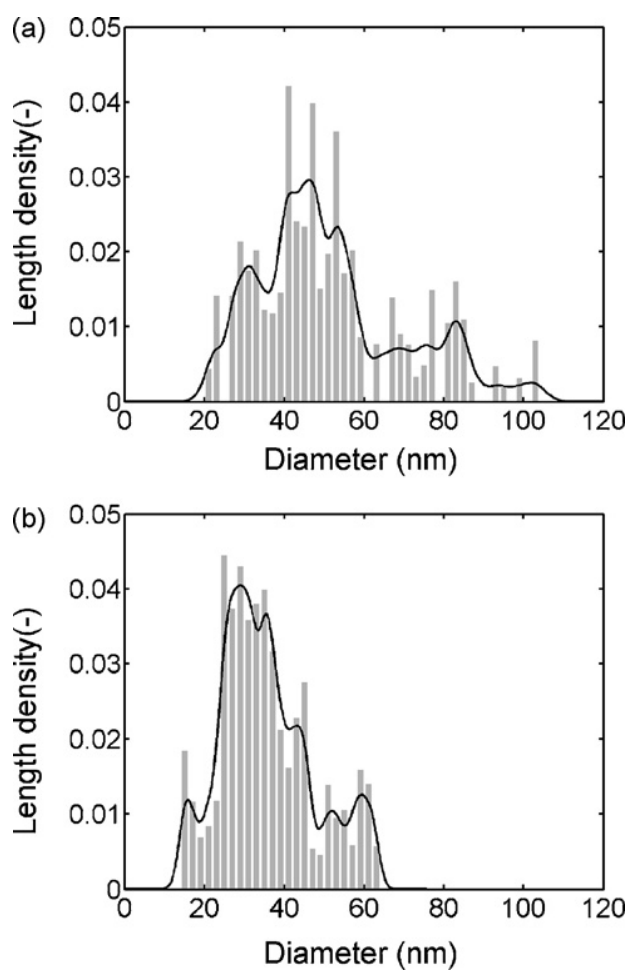

Fig. 3. Length density distributions of nanotubes in samples CNT-2 (a) and CNT-3 (b) as a function of their outer diameter; the solid line was obtained from Eq. (1).

renders the distributions independent of the binning of the data. Moreover, this method takes explicitly account of the error $\sigma$ on the measurement of a single nanotube. For both samples, the distributions are roughly bimodal. In the case of CNT-2, a first group of small nanotubes, with a diameter between 20 and $60 \mathrm{~nm}$, corresponds to the two branches of the Y-junctions (e.g. 55 and $44 \mathrm{~nm}$ in Fig. 2b). The second group, with larger diameters between 60 and $100 \mathrm{~nm}$, corresponds to the main branch of the Y-junction (e.g. $76 \mathrm{~nm}$ in Fig. 2b). Similarly, the diameter distribution of CNT3 is also bimodal, with the first group corresponding to the two branches (e.g. 35 and $40 \mathrm{~nm}$ in Fig. 2c), and the second group corresponding to the main branch (e.g. $61 \mathrm{~nm}$ in Fig. 2c).

The question arises about the mechanism by which these structures form. Quite generally, carbon nanotubes can form either via a root-growth or tip-growth mechanism [16]; the observations of the present paper suggest a tip-growth mechanism [17]. In the frame of a tip-growth, Y-junctions can occur either by the splitting of a large metal particle into two smaller ones during the tip-growth of the main branch of the Y (Fig. 4b), or from the merger of the
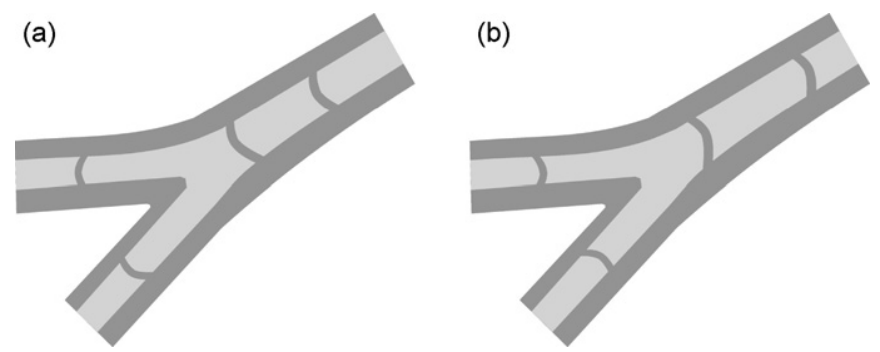

Fig. 4. Two possible concavities of the inner structures of the tubes, corresponding to two different mechanisms of formation of the Y-junctions in the frame of a tipgrowth scenario: (a) two metal particles merge into a larger particle and (b) a single metal particle splits into two smaller particles.

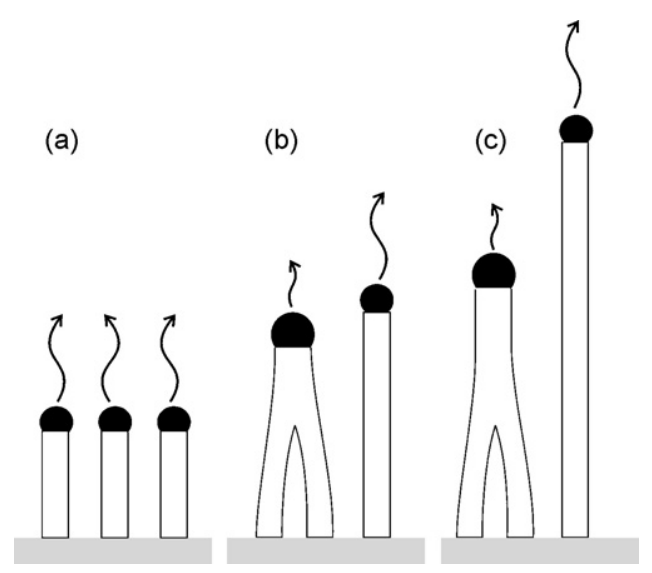

Fig. 5. Proposed mechanism for the formation of Y-junctions in carbon nanotubes arrays: (a) all nanotubes are initially identical, they therefore grow at the same rate and their metal particles are close to each other; (b) a Y-junction is formed when two particles touch and merge; (c) when the growth continues afterwards, the growth rate are no longer necessarily the same; the metal particles move therefore apart from each other and the merging becomes less frequent.

two small particles at the top of the two branches of the Y (Fig. 4a). The orientation of the concavity of the inner walls in Fig. 2d (see arrows) proves that the structure formed through the progression of the metal particle from the right to the left. This means that the structure has formed by the merger of the two branches of the Y, rather than by the splitting of its main branch. This scenario is also supported by Fig. 2a that shows that the metallic particle in one of the branches was moving towards the junction when it stopped.

There might be many different mechanisms of formation of Y-junctions according to the experimental conditions; the mechanism reported here is not necessarily the same as in other nanotubes [4-7] in which bamboo-like structures are not observed. In the frame of the proposed merger scenario, the occurrence of Y-junctions is proportional to the probability of two particles to meet during the growth of the nanotubes. Looking at Fig. 1, it can be surmised that the aligned growth of nanotubes maximizes the probability of touching-and-merging of the metal particles, as illustrated in Fig. 5.

\section{Conclusions}

TEM observation suggests that Y-junctions are common in multi-walled carbon nanotubes arrays obtained by catalytic chemical vapor deposition of iron phtalocyanine. Furthermore, the diameter distributions of the samples are found to be bimodal; one mode corresponds to the two branches of the Y, and the other mode corresponds to its main trunk. The orientation of the concavity of inner structures of the nanotubes shows that the observed Y-junctions form by the merger of metal particles during a tipgrowth scenario of the tubes. From the present analysis, the same process is expected in any situation in which aligned nanotubes are formed via a tip-growth mechanism.

\section{Acknowledgments}

Part of this work was done during the stay of DZ in Belgium, supported by the ALFA Program of the E.U. (project ALFA II 0412 FA FI). CJG is grateful to the Belgian National Fund for Scientific Research (FNRS) for a postdoctoral researcher position. DZ and ALC acknowledge financial support from CONICET, UBA, and ANPCYTFONCYT from Argentina. 


\section{References}

[1] M. Monthioux, V.L. Kuznetsov, Who should be given the credit for the discovery of carbon nanotubes? Carbon 44 (2006) 1621-1623.

[2] T.W. Ebbesen, H.J. Lezec, H. Hiura, J.W. Bennett, H.F. Ghaemi, T. Thio, Electrical conductivity of individual carbon nanotubes, Nature 382 (1996) 54-56.

[3] P.R. Bandaru, Electrical characterization of carbon nanotube Y-junctions: a foundation for new nanoelectronics, J. Mater. Sci. 42 (2007) 1809-1818.

[4] B.C. Satishkumar, P.J. Thomas, A. Govindarai, C.N.R. Rao, Y-junction carbon nanotubes, Appl. Phys. Lett. 77 (2000) 2530-2532.

[5] W.Z. Li, B. Pandey, Y.Q. Liu, Growth and structure of carbon nanotube Yjunctions, J. Phys. Chem. B 110 (2006) 23694-23700.

[6] H. Zhu, L. Ci, C. Xu, J. Liang, D. Wu, Growth mechanism of Y-junction carbon nanotubes, Diamond Relat. Mater. 11 (2002) 1349-1352.

[7] W.Z. Li, J.G. Wen, Z.F.S. Ren, Straight carbon nanotube Y junctions, Appl. Phys. Lett. 79 (2001) 1879-1881.

[8] A.N. Andriotis, M. Menon, D. Srivastava, L. Chernozatonskii, Rectification properties of carbon nanotube "Y-Junctions", Phys. Rev. Lett. 87 (2001), 066802-1-4.

[9] J.-M. Ting, C.-C. Chang, Multijunction carbon nanotube network, Appl. Phys. Lett. 80 (2002) 324-325

[10] S. Huang, L. Dai, A.W.H. Mau, Patterned growth and contact transfer of wellaligned carbon nanotube films, J. Phys. Chem. B 103 (1999) 4223-4227.
[11] D.A. Zilli, P.R. Bonelli, A.L. Cukierman, in: X. Huang (Ed.), Nanotechnology Research: New nanostructures, nanotubes and nanofibers, Nova Science Publishers, New York (2008).

[12] D.A. Zilli, P.R. Bonelli, A.L. Cukierman, Effect of alignment on adsorption characteristics of self-oriented multi-walled carbon nanotube arrays, Nanotechnology 17 (2006) 5136-5141.

[13] C. Gommes, S. Blacher, K. Masenelli-Varlot, C. Bossuot, E. McRae, A. Fonseca, J. B'Nagy, J.-P. Pirard, Image analysis characterization of multi-walled carbon nanotubes, Carbon 41 (2003) 2561-2572.

[14] E. Parzen, On estimation of a probability density function and mode, Ann. Math. Stat. 33 (1962) 1065-1076.

[15] W. Liu, N.N. Clark, A.I. Karamavruc, General method for the transformation of chord-length data to a local bubble-size distribution, AIChE J. 42 (1996) 2713-2720.

[16] C.-C. Chuang, W.-L. Liu, W.-J. Chen, J.-H. Huang, Temperature and substrate dependence of structure and growth mechanism of carbon nanofiber, Appl. Surf. Sci. 254 (2008) 4681-4687.

[17] S. Helveg, C. López-Cartes, J. Sehested, P.L. Hansen, B.S. Clausen, J.R. RostrupNielsen, F. Abild-Pedersen, J.K. Nørskov, Atomic-scale imaging of carbon nanofibre growth, Nature 427 (2004) 426-429. 